\title{
Indicaciones actuales de colocación de stent uretral Allium - revisión de la literatura
}

\section{Current Indications for Urethral Allium Stent Placement-Review of the Literature}

\author{
Matilde Henao ${ }^{1}$ Federico Gaviria ${ }^{2}$
}

Address for correspondence Matilde Henao, MR, Residente de Urología, Universidad CES, Medellín/Colombia

(e-mail: matilde_henao@hotmail.com; matildehenao91@gmail.com).

Urol Colomb 2019;28:204-208.

\section{Resumen \\ Palabras Clave \\ - stent \\ - uretral \\ - Allium \\ - estenosis \\ - estrechez \\ - uretra \\ - reconstructiva \\ - urología}

\begin{abstract}
Urethral stricture disease is a pathology with a prevalence of $0.6 \%$ of the population The gold standard established for its management is urethroplasty with variable success rates of up to $90 \%$ to $95 \%$.

The urethral stent arose in 1988 manufactured with stainless steel mesh. The results obtained showed the severe complications of its implantation. In 2008, the Allium
\end{abstract}

La estrechez de la uretra es una patología con una prevalencia del 0,6\% de la población El estándar de oro establecido para su manejo es la uretroplastia con tasas de éxito variables de hasta el $90 \%$ al $95 \%$.

El stent uretral surgió en 1988 fabricado con malla de acero inoxidable. Los resultados obtenidos demostraron las complicaciones severas de su implantación. En el 2008 se introdujo el stent Allium producido en Israel con propiedades diferentes como elasticidad y flexibilidad. Puede utilizarse en diferentes entidades como estrechez de uretra bulbar,hiperplasia de próstata y la estenosis de la unión uretrovesical. Recientemente este Stent se está comercializando en Colombia, por lo cual consideramos importante revisar la literatura mundial, las complicaciones e indicaciones reales y así intentar evitar el uso inapropiado de esos dispositivos.

Realizamos una Revisión de la literatura en PubMed y ClinicalKey incluyendo los términos, "Stent", "Allium" y "Urethral", incluyendo articulos en inglés y en español. Se encontraron 4 artículos retrospectivos en poblaciones heterogéneas con tasas de éxito inferiores a la cirugía reconstructiva. No se encontraron estudios comparativos. Las indicaciones actuales para colocar un stent uretral podrían ser pacientes en quienes no se puede realizar uretroplastia, o en quienes el manejo endoscópico de la uretra posterior no haya sido efectivo. También puede ser una alternativa para pacientes con sonda vesical permanente por hiperplasia prostática no aptos para cirugía.

received

May 9, 2018

accepted

July 30, 2018
DOI https://doi.org/

10.1055/s-0038-1669932.

ISSN 0120-789X.

eISSN 2027-0119.
Copyright (c) 2019, Sociedad Colombiana License terms de Urología. Publicado por Thieme Revinter Publicações Ltda., Rio de Janeiro, Brazil. Todos los derechos reservados. 

Keywords
- stent
- urethral
- allium
- stenosis
- stricture
- urethra
- reconstructive
- urology

stent was produced in Israel with different properties such aselasticity and flexibility. It can be used in different entities such as bulbar urethra strictures, prostatic hyperplasia and stenosis of the urethrovesical junction. Recently this Stent is being marketed in Colombia, which is why we consider important to review the literature, complications and real indications and thus try to avoid the inappropriate use of these devices.

We conducted a literature review in PubMed and ClinicalKey including the terms "Stent", "Allium" and "Urethral", including articles in English and Spanish. We found 4 retrospective articles in heterogeneous populations with success rates lower than reconstructive surgery. No comparative studies were found.

Current indications to place a stent urethral could be patients in whom urethroplasty cannot be performed, or in patients in which endoscopic management of the posterior urethra has not been effective. It can also be an alternative for patients with permanent bladder catheter due to prostatic hyperplasia not suitable for surgery.

\section{Introducción}

La estrechez de la uretra es una patología con una prevalencia del $0,6 \%$ de la población, ${ }^{1}$ y se considera una enfermedad de alto impacto debido a la alteración en la calidad de vida del paciente, la predisposición a infecciones del tracto urinario, retención urinaria, litiasis y enfermedad renal crónica.

Puede ser causada por trauma, condiciones inflamatorias, iatrogenia y tratamiento para la hiperplasia y el cáncer de próstata.

El estándar de oro establecido para su manejo es la uretroplastia con tasas de éxito variables de hasta el $90 \%$ al $95 \%^{2}$

En casos seleccionados, se prefiere la uretrotomía interna endoscópica (estenosis de la unión uretrovesical, estenosis membranosas o bulbares cortas $<1 \mathrm{~cm}$ y laxas) $\sin$ espongiofibrosis y no de origen traumático. ${ }^{2}$

\section{Evolución histórica}

El concepto de stent uretral se contempla desde 1969 sin embargo, fue descrito en 1988 como una adaptación de los stent endovasculares (UroLume Urethral Stent (American Medical Systems) fabricados con malla de acero inoxidable (-Fig. 1).

Los resultados obtenidos a largo plazo con ese tipo de Stent demostraron las dificultades y complicaciones severas de su implantación., 3

Incluyendo reintervención, migración en el $45 \%$, hiperplasia obstructiva intra-stent en el $32 \%$, estrechez $25 \%$, incrustación o calcificación en el $17 \%$, infección del tracto urinario en el $27 \%$, hematuria, dolor e incontinencia. ${ }^{5}$

La reintervención de esos pacientes genera un reto para el urólogo y la cirugía reconstructiva, requiriendo extracción del stent y uretroplastia posterior con baja tasa de éxito y necesidad de procedimientos posteriores en un $50 \%{ }^{6}$

Debido a esas complicaciones dejó de ser producido por American Medical Systems y no se coloca en la actualidad.

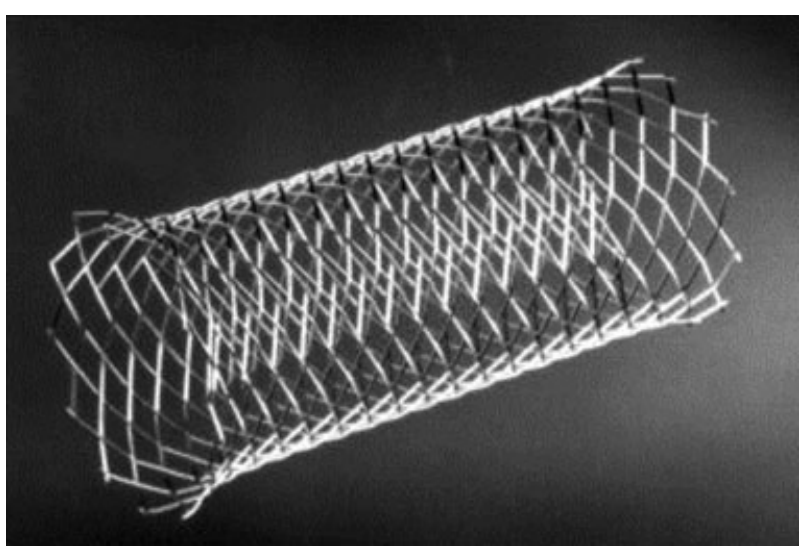

Fig. 1 Adaptación de los stent endovasculares (UroLume Urethral Stent (American Medical Systems) fabricados con malla de acero inoxidable.

En el 2008 se introdujo el stent Allium producido en Israel con propiedades diferentes a los stent previos, debido a que es elástico y altamente flexible.

Es fabricado con nitinol y cubierto con un copolimero que previene el crecimiento de tejido interno y reduce la incrustación, formación de cálculos y calcificaciones, aunque esas complicaciones están descritas. ${ }^{7}$

Recientemente este Stent está siendo comercializando en Colombia, por lo cual consideramos importante revisar la literatura mundial, las complicaciones e indicaciones reales y así intentar evitar el uso inapropiado de esos dispositivos.

Otra ventaja es que el stent permite retirarse de manera sencilla debido a su capacidad de desenrrollarse para retiro endoscópico lo cual lo diferencia de los stent previos (- Fig 2).

Existen diferentes tipos de Stent de Allium (BUS, Bulbar urethral Stent) (TPS, Triangular prostatic stent), (RPS, Round posterior Stent), para utilizarlo en diferentes entidades como la estrechez de uretra bulbar, la hiperplasia de próstata y la estenosis de la unión uretrovesical respectivamente (-Figs. $3 \& 4$ ).

En cuanto a la técnica quirúrgica vienen diseñados para colocarse a través de una pistola que permite introducir un lente y visualizar la uretra para lograr la colocación del stent 

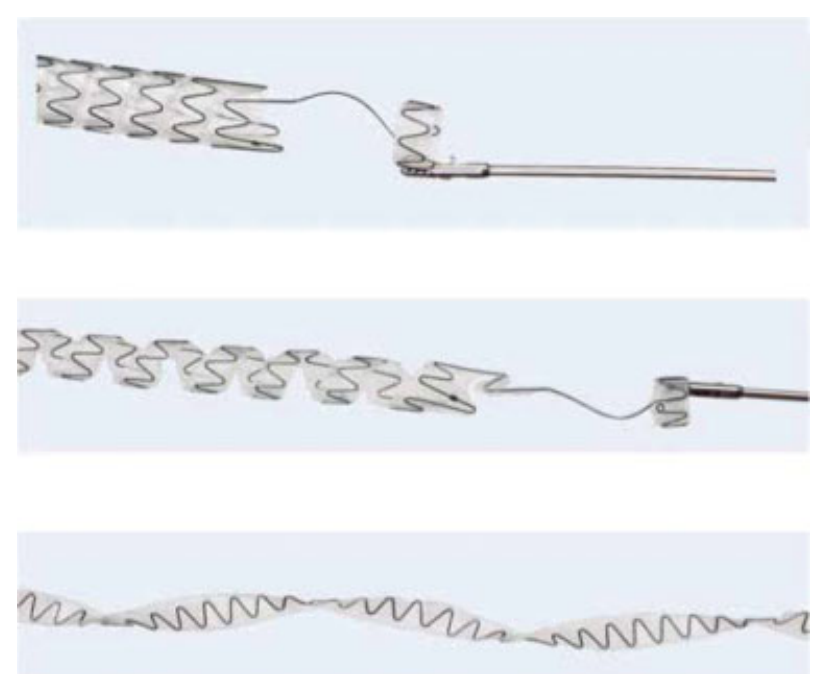

Fig. 2 Capacidad de desenrrollarse para retiro endoscópico.

en el lugar deseado, además tiene un mecanismo para expandir el stent y fijarlo a la uretra (-Fig. 5).

\section{Materiales y Métodos}

Se realizó una Revisión de la literatura en PubMed y ClinicalKey incluyendo los términos, "Stent", "Allium" y "Urethral", incluyendo artículos en inglés y en español, se incluyeron artículos que evaluaran la aplicación de los stent uretrales allium.

Se encontraron 4 artículos que evaluaran la aplicación de esos stent de carácter retrospectivo.

No se encontraron estudios que lo comparen con el estándar de oro (la uretroplastia perineal y el manejo endoscópico).

El Allium BUS (Bulbar Urethral Stricture) está diseñado para las estrecheces de la uretra bulbar, viene precargado en un instrumento de 22Fr y se expande hasta 45Fr luego de liberarlo, su uso está diseñado para 12 meses, no tiene mecanismo de anclaje.

En un estudio realizado en turquía, ${ }^{8}$ se incluyeron 28 pacientes desde el 2010 a 2014, los criterios de inclusión eran pacientes con estrechez bulbar recurrente con al menos 2 dilataciones previas o uretrotomías internas endoscópicas y los criterios de exclusión eran la presencia de estrechez peneana o estenosis de la uretra posterior. Los pacientes tenían en promedio 62 años y la etiología más frecuente de la estrechez era la iatrogenia en un $36 \%$ seguido del trauma en un $25 \%$. Durante el procedimiento quirúrgico se realizó uretrotomía previo a la inserción del stent. El tiempo quirúrgico promedio fue de 15 a 35 minutos.

Todos los stent se retiraron entre el tercer y sexto mes. El seguimiento se realizó con uroflujometría y residuo postmiccional a los 3, 6 y 12 meses posterior al retiro del stent y luego anual para un seguimiento promedio de 29 meses.

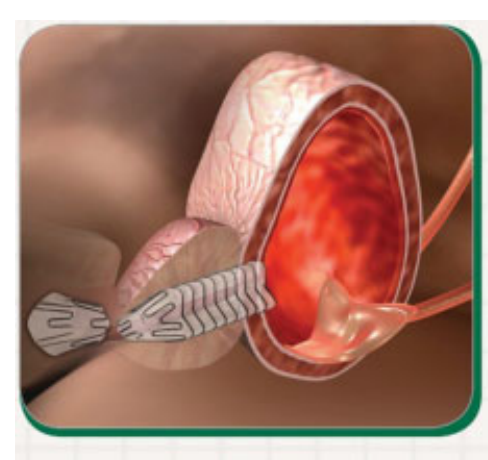

TPS

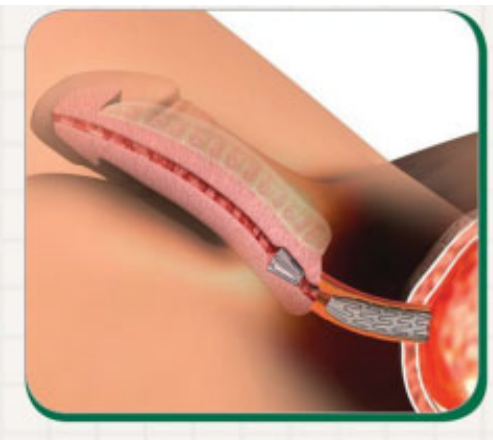

RPS

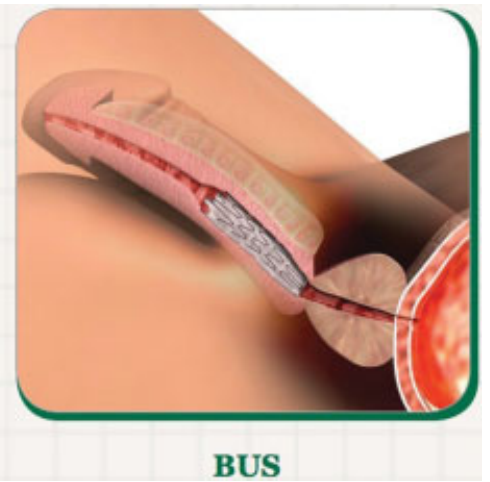

BUS

Fig. 3 Ilustración de tipos de Stent de Allium aplicados: BUS, bulbar urethral stent; TPS, triangular prostatic stent; RPS, round posterior stent.
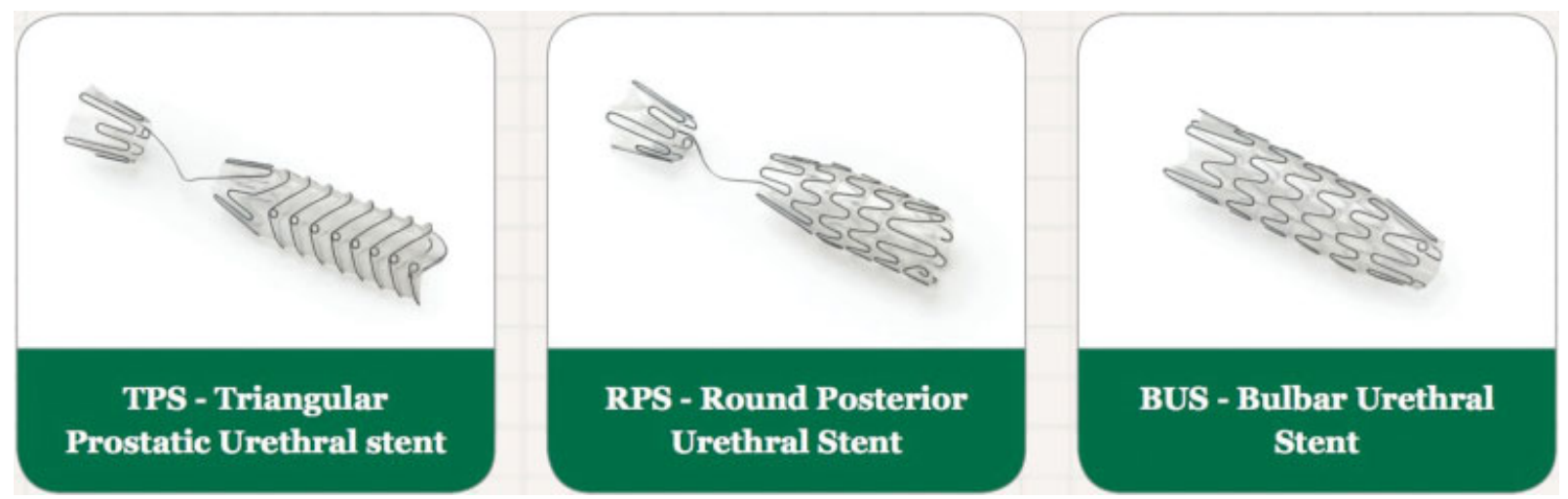

Fig. 4 Ilustración de tipos de Stent de Allium: BUS, bulbar urethral stent; TPS, triangular prostatic stent; RPS, round posterior stent. 


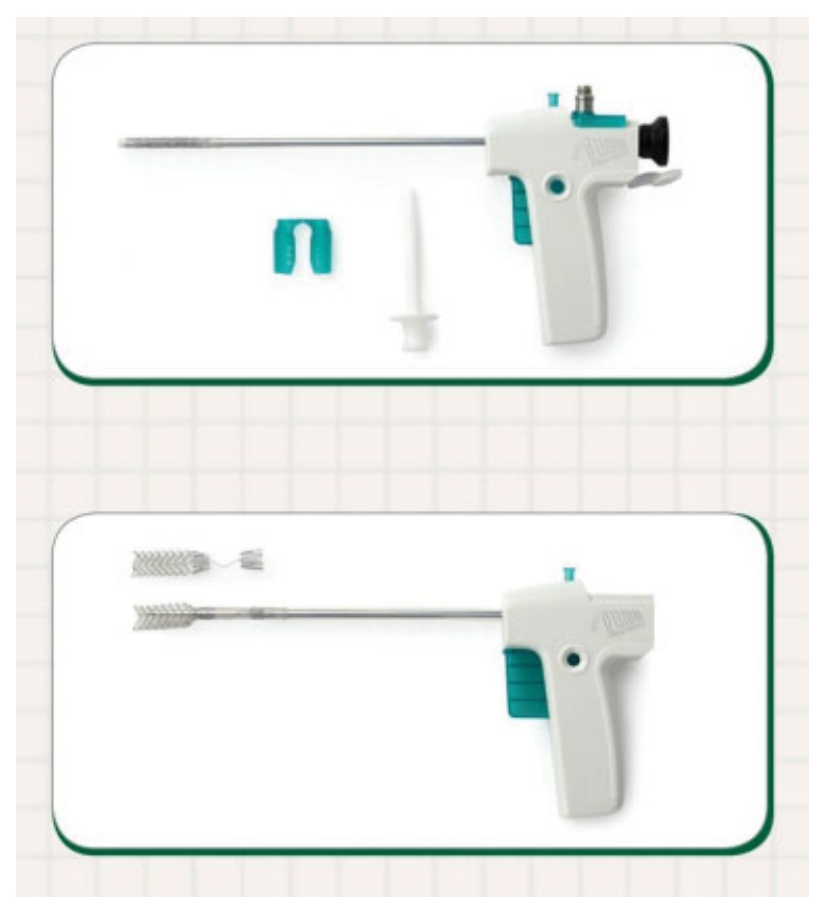

Fig. 5 Pistola que permite introducir un lente y visualizar la uretra para lograr la colocación del stent.

Reportan una tasa de éxito del $64,2 \%$ definida como ausencia de recurrencia de estrechez.

En otro estudio realizado también en Turquía de 2009 a $2012^{7}$ se colocaron 54 stent BUS, con estrecheces de la uretra bulbar de en promedio $2,44 \mathrm{~cm}$, se retiraron entre los 3 y 18 meses siguientes, seguimiento promedio luego de retiro de stent de 8,3 meses, los criterios de éxito fueron ausencia de recurrencia de estrechez de uretrografía o cistoscopia, Qmax mayor a $15 \mathrm{~mL} / \mathrm{seg}$, encontrando una tasa de éxito del $75 \%$ a 2 años.

El Allium TPS (Triangular Prostatic Stent), está diseñado para pacientes con hiperplasia prostática no aptos para llevar a cirugía, diseñado de forma triangular para acomodarse a la uretra prostática con un alambre transesfinteriano para intentar preservar la continencia al no incluir el esfínter externo en la área del stent. Ese tipo de stent incluye un mecanismo de anclaje para evitar la migración.

En un estudio realizado en Turquía e Israel entre el 2008 $2014^{9}$ se evaluaron de manera retrospectiva 51 pacientes con implantación de este stent que no pudieran ser llevados a prostatectomía por sus comorbilidades con clasificación de ASA de 3 y 4.

El seguimiento se realizó a 12 meses evaluando como desenlace primario mejoría del IPSS y en flujo máximo. Los pacientes tenían 72 años en promedio y volumen prostático de $38 \mathrm{gr}$ en promedio, las complicaciones más frecuentes fueron el dolor en el 18\% que resolvió al quinto día postoperatorio y la infección en el $9 \%$.

Dos pacientes requirieron extracción del stent, uno debido a hematuria intratable y otro a infección crónica.

No ocurrió migración, incontinencia u oclusión del stent durante el tiempo de seguimiento. El puntaje de IPSS se redujo de 26 a 7,7 y el flujo máximo aumento de $5,5 \mathrm{~mL} / \mathrm{seg}$. a $16 \mathrm{~mL} / \mathrm{seg}$. Aunque los resultados fueron favorables, este estudio incluye pocos pacientes con un seguimiento corto.

El Allium RPS (Round posterior urethral stent) está diseñado para estenosis de la unión uretrovesical luego de prostatectomía radical o de estenosis de la uretra posterior, también cuenta con el mecanismo de alambre transesfinteriano.

En un estudio preliminar en Malaga, España, ${ }^{10}$ se evaluaron 10 pacientes, 7 con estenosis posterior a la prostatectomía radical, uno con HIFU (High intensity focused ultrasound), uno con fractura pélvica y otro con estenosis iatrogénica.

Los pacientes tuvieron el stent en promedio 5,5 meses, en 2 pacientes ocurrió migración del stent. En el momento están pendientes los resultados de eficacia del stent en este estudio.

En la literatura no está establecido cuál es el tiempo óptimo en el cual se debe retirar el stent, ni si su colocación debe ser sucesiva vs única.

No existen otros estudios que evalúen su eficacia en la literatura.

\section{Recomendaciones}

Las guías americanas del 2016 no recomiendan el uso de stent uretral. $^{11}$

Las guías europeas del 2014 recomiendan no utilizar el stent uretral en pacientes con estrechez de origen traumático y en pacientes que son candidatos para uretroplastia, con un grado de recomendación A.

Solo recomiendan considerarlo en pacientes con estrecheces cortas que no son candidatos por sus comorbilidades a uretroplastia con grado de Recomendación $\mathrm{B}^{2}$

Las indicaciones reales en la actualidad para la colocación de un stent uretral son en una subpoblación de pacientes en los cuales no se puede realizar uretroplastia por su condición de base, o en quienes el manejo endoscópico con uretrotomía interna endoscópica y dilatación de la uretra posterior no haya sido efectiva y no sea candidato a cirugía reconstructiva.

También puede considerarse como alternativa para pacientes con sonda vesical permanente por hiperplasia prostática no aptos para cirugía.

No se debe utilizar en pacientes en los cuales la etiología de la estrechez es el trauma ni en quienes la opción ideal es la uretroplastia por lo cual esta continúa siendo el manejo quirúrgico estándar que provee las mejores tasas de éxito realizado por cirujanos experimentados.

Los estudios clínicos reportados en la literatura son de carácter retrospectivo, escasos y con bajo número de pacientes, con tasas de éxito significativamente inferiores a la cirugía reconstructiva (entre el $60 \%$ y el $75 \%$ ), y en el presente pocas instituciones lo utilizan de manera rutinaria.

En caso de que el urólogo que evalúa al paciente con estrechez de uretra no cuente con la experiencia necesaria para abordar los casos, en lugar de utilizar el stent como primera medida, debe remitir el paciente a urólogos que tengan alto volumen y experiencia con la uretroplastia para proporcionarle la mayor probabilidad de un buen desenlace quirúrgico al paciente. 


\section{Conclusiones}

Se debe seleccionar el paciente en el cual exista una indicación real y no pretender remplazar la cirugía reconstructiva.

Se requieren estudios clínicos con mayor número de pacientes, con la indicación adecuada y un seguimiento a largo plazo para poder recomendar su uso de manera rutinaria.

Protección de personas y animales

Los autores declaran que para esta investigación no se han realizado experimentos en seres humanos ni en animales.

\section{Confidencialidad de los datos}

Los autores declaran que en este artículo no aparecen datos de pacientes.

Derecho a la privacidad y consentimiento informado Los autores declaran que en este artículo no aparecen datos de pacientes.

\section{Referencias}

1 Santucci RA, Joyce GF, Wise M. Male urethral stricture disease. J Urol 2007;177(05):1667-1674
2 SIU/ICUD Consultation on Urethral Strictures: Dilation, Internal Urethrotomy, and Stenting of Male Anterior Urethral Strictures, UROLOGY 83: S18eS22, 2014

3 De Vocht TF, van Venrooij GE, Boon TA. Self-expanding stent insertion for urethral strictures: a 10-year follow-up. BJU Int 2003;91(07):627-630

4 J Urol. 1996 Mar;155(3):904-8. Long-term results of urolume urethral stent for recurrent urethral strictures

5 Hussain M, Greenwell TJ, Shah J, Mundy A. Long-term results of a self-expanding wallstent in the treatment of urethral stricture. BJU Int 2004;94(07):1037-1039

6 Chapple CR, Bhargava S. Management of the failure of a permanently implanted urethral stent-a therapeutic challenge. Eur Urol 2008;54(03):665-670

7 Culha M, Ozkuvanci U, Ciftci S, et al. Management of recurrent bulbar urethral stricture-a 54 patients study with Allium bulbar urethral stent (BUS). Int J Clin Exp Med 2014;7(10):3415-3419

8 Temeltas G, Ucer O, Yuksel MB, Gumus B, Tatli V, Muezzinoglu T. The long-term results of temporary urethral stent placement for the treatment of recurrent bulbar urethral stricture disease? Int Braz J Urol 2016;42(02):351-355

9 Allium TM, Yildiz G, Bahouth Z, et al. TPS-A New Prostatic Stent for the Treatment of Patients with Benign Prostatic Obstruction: The First Report. J Endourol 2015

10 A new self expanding, large caliber Allium Bladder neck stent (RPS) in posterior urethral or urethrovesical anastomotic stenosis, Preliminary results

11 MALE URETHRAL STRICTURE. AUA GUIDELINE, American Urological Association (AUA) 2016 